\title{
Saturation Studies of the E-Beam Sustained Discharge Atomic Xenon Laser
}

\author{
H. Botma, P. J. M. Peters, and W. J. Witteman
}

\begin{abstract}
In an electron beam sustained discharge Xenon laser the discharge energy deposition has been varied in order to investigate the saturation effect on the Xenon laser. The current density of the electron beam is varied separately in the range of 0.1-2.7 $\mathrm{A} / \mathrm{cm}^{2}$ to obtain optimized discharge excitation conditions as a function of electron beam current density and gas pressure. An optimal fractional ionization $f=3.5-4 \times$ $10^{-5}$ is found, independent of the electron beam parameters. The synergy of electron beam and discharge excitation has resulted in a maximum specific energy of $15 \mathrm{~J} / 1$ at a total gas pressure of 9 bar.
\end{abstract}

\section{INTRODUCTION}

T $\mathrm{HE}$ atomic Xenon laser is a promising alternative to the $\mathrm{CO}_{2}$ laser. Though the maximum specific energy of an electron-beam ( $e$-beam) sustained discharge xenon laser is $15 \mathrm{~J} / 1$ [this work] compared to $60 \mathrm{~J} / 1$ for an $e$-beam sustained discharge $\mathrm{CO}_{2}$ laser [1], its brightness can be nine times higher because of the shorter wavelength.

Atomic Xenon lasers are operated using several excitation techniques, among which are: pure electron beam excitation [2]-[5], electron beam sustained discharge excitation [6]-[10], fast discharge excitation [11] and X-ray preionized discharge excitation [12]. All these techniques also have been successfully applied to excimer laser studies in the past [13]. Some authors [14]-[16] studied the impact of different $e$-beam current densities on the efficiency and laser output energy. In a previous paper [6], we investigated the essential role of the $e$-beam on the laser performance. In Fig. 1, the xenon energy level diagram is given. From this figure, it can be seen that the xenon laser oscillates at five eye-safe infrared transitions; the $1.73 \mu \mathrm{m}$ transition carries over $90 \%$ of the laser energy in argon based mixtures.

In this work, the influence of the $e$-beam current density and the $e$-beam energy on the laser output and on the discharge characteristics will be investigated. Two different $e$-beam energies will be used while the current density of each $e$-beam is changed by using partially transmitting masks.

Manuscript received November 16, 1992. This work was supported in part by the Netherlands Technology Foundation (STW).

The authors are with the University of Twente, Department of Applied Physics, 7500 AE Enschede, The Netherlands.

IEEE Log Number 9211365.



Fig. 1. The Xenon energy level diagram.

\section{Experimental Configuration}

The experiments are carried out using the laser head described in [6]. Electrons produced by field emission from a cold cathode are accelerated in a vacuum diode by a high voltage pulse produced by a Marx generator and enter the laser head through a $25-\mu \mathrm{m}$ thin titanium foil. The foil is supported by a Hibachi structure which limits the excitation area to $46 \times 5 \mathrm{~cm}^{2}$. In the present experiments we want to vary the average $e$-beam current density without changing the electron energy. For that reason masks are placed between the foil and the active volume. The masks have transmission factors of $9 \%, 16 \%$, and $33 \%$. The insertion of the masks reduces the gap width (Table I) thus specific energies (averaged over the volume) will be used.

Two different $e$-beam parameters are used: a $180 \mathrm{keV}$ $e$-beam with an unmasked peak current density of 1.2 $\mathrm{A} / \mathrm{cm}^{2}$ and a total duration of $0.9 \mu \mathrm{s}$ and a $270 \mathrm{keV}$ $e$-beam with an unmasked peak current density of 2.7 $\mathrm{A} / \mathrm{cm}^{2}$ having a duration of $1.2 \mu \mathrm{s}$. For convenience, these $e$-beams will be referred to by using the subscripts lo and hi, respectively. The $e$-beam current density is measured by using the discharge electrode as one large Faraday cup with an effective area of $46 \times 5 \mathrm{~cm}^{2}$, connected to ground by a measuring resistance $\mathbf{R}=0.11 \Omega$. To avoid scattering of the $e$-beam electrons to the walls during the measurements the laser cell has to be evacuated to a pressure below $10^{-3}$ mbar. Alternatively, the laser cell can be filled with helium as no influence of the helium pressure on the measured $e$-beam current is observed in strong contrast with the use of argon. 
TABLE I

Discharge Dimensions and Current Densities for the Various Masks

\begin{tabular}{lccc}
\hline & $\begin{array}{c}\text { Gap Width } \\
{[\mathrm{cm}]}\end{array}$ & $\begin{array}{c}j_{\mathrm{lo}} \\
{\left[\mathrm{A} / \mathrm{cm}^{2}\right]}\end{array}$ & $\begin{array}{c}j_{\mathrm{hi}} \\
{\left[\mathrm{A} / \mathrm{cm}^{2}\right]}\end{array}$ \\
\hline $9 \%$ mask & 1.20 & 0.11 & 0.24 \\
$16 \%$ mask & 1.20 & 0.19 & 0.43 \\
$33 \%$ mask & 1.35 & 0.40 & 0.90 \\
no mask & 2.00 & 1.20 & 2.70
\end{tabular}

During our laser experiments the laser cell is filled with a 99.6:0.4 argon-xenon mixture at pressures up to 9 bar. The optical cavity consists of one flat $\mathrm{ZnSe}$ output coupler with $50 \%$ reflectance and one totally reflecting gold mirror with a radius of curvature of $7.5 \mathrm{~m}$, the optical aperture is $5.9 \mathrm{~cm}^{2}$. The sustainer circuit consists of a capacitor $C=5.4 \mu \mathrm{F}$ in series with a $\mathrm{NaCl}$-solution resistor $R$ $=0.05 \Omega$. This capacitor is switched to the discharge electrode $1 \mu$ s before the start of the $e$-beam by one spark gap. The self inductance $L$ of this discharge circuit is approximately $100 \mathrm{nH}$.

InAs photo diodes type $\mathrm{J} 12-18 \mathrm{c}$ are used to detect the laser radiation. A CdTe window in front of one photo diode transmits all five infrared transitions and blocks all visible light. A narrow band dielectric filter at $1.73 \mu \mathrm{m}$ transmits only that specific line to a second photo diode. In this way the time evolutions of the total (all-line) laser intensity, the laser intensity of the $1.73-\mu \mathrm{m}$ line and discharge voltage and current are measured by Philips PM3350 and PM3355 digitizers. The all-line photo diode waveform area equals the laser energy apart from a calibration factor. This factor is found by comparing the waveform area to the laser energy measured with an ED 500 Gentec Joule meter.

\section{Experimental Results}

\section{A. Laser Output Energy}

The first part of our investigation is concerned with the dependency of the laser output energy on the $e$-beam current density. At each combination of $e$-beam energy and current density the deposited discharge energy is increased by increasing the charge voltage of the sustainer capacity at several gas densities until saturation of the laser output energy is observed. The deposited discharge energy is found by calculating the integral of the discharge electrode voltage times the discharge current.

In Figs. 2-5 the specific laser output energy is plotted against this discharge energy for a gas pressure $P$ of 3,5 , 7 , and 9 bar, respectively. In Fig. 2, two pairs of almost coinciding curves are apparent. These curves show that at $\mathbf{P}=3$ bar the laser operation depends only on the $e$-beam current density, not on the $e$-beam energy: The effective current densities are almost equal for each pair. The lowest pair of curves has an $e$-beam current density of about $0.4 \mathrm{~A} / \mathrm{cm}^{2}$ just behind the foil. Increasing $j$ to about 1 $\mathrm{A} / \mathrm{cm}^{2}$ results in the upper pair of curves. Apparently, the laser output energy is independent of the kinetic energy of the incoming electrons.

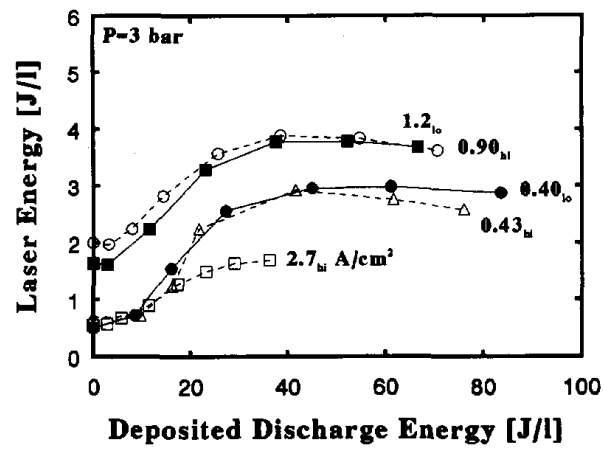

Fig. 2. Laser energy versus discharge energy at 3 bar using two different $e$-beams (filled markers: $e$-beam - $_{\mathrm{lo}}$, open markers: $e$-beam hi $_{\mathrm{h}}$, numbers: $\mathrm{A} / \mathrm{cm}^{2}$ )

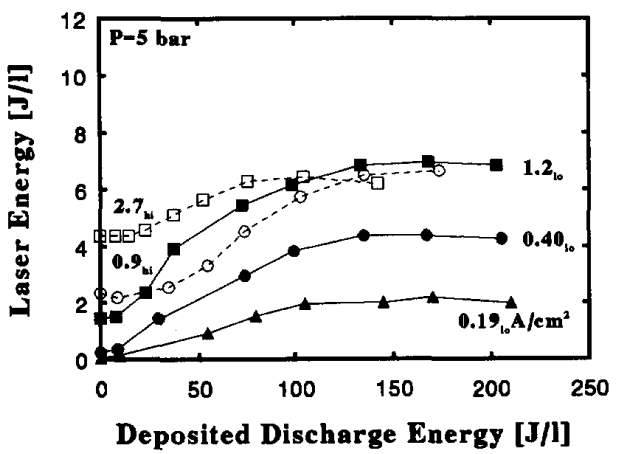

Fig. 3. Laser energy at 5 bar, conditions as in Fig. 2.

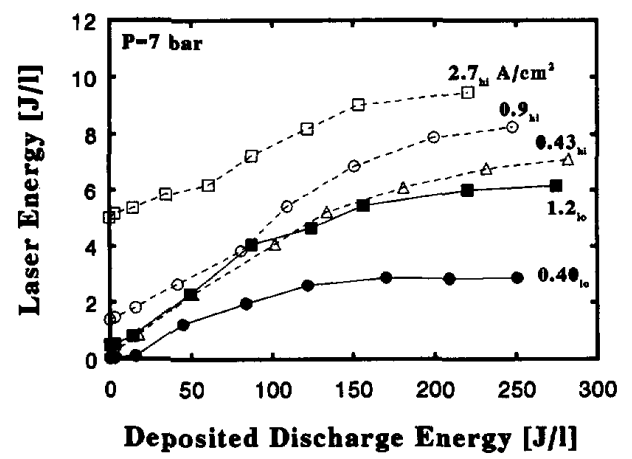

Fig. 4. Laser energy at 7 bar, conditions as in Fig. 2.

For pure $e$-beam excitation a further increase of $j$ to 2.7 $A / \mathrm{cm}^{2}$ reduces the specific laser output energy considerably from $2.0 \mathrm{~J} / 1$ to $0.5 \mathrm{~J} / 1$. This decrease is caused by Electron Collison Mixing (ECM, [2], [3]) of the upper and lower laser levels by $e$-beam electrons. Nevertheless, the addition of an electrical discharge under these high $e$-beam current density circumstances increases the specific output energy of the laser to $1.5 \mathrm{~J} / 1$. To illustrate this point laser output power waveforms are presented in Figs. 6 and 7. The lowest (dotted) waveform in Fig. 6

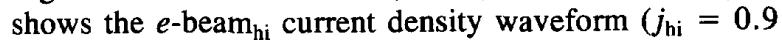
$\mathrm{A} / \mathrm{cm}^{2}$ ). The next higher waveform shows the laser power 




Deposited Discharge Energy [J/l]

Fig. 5. Laser energy at 9 bar, conditions as in Fig. 2.

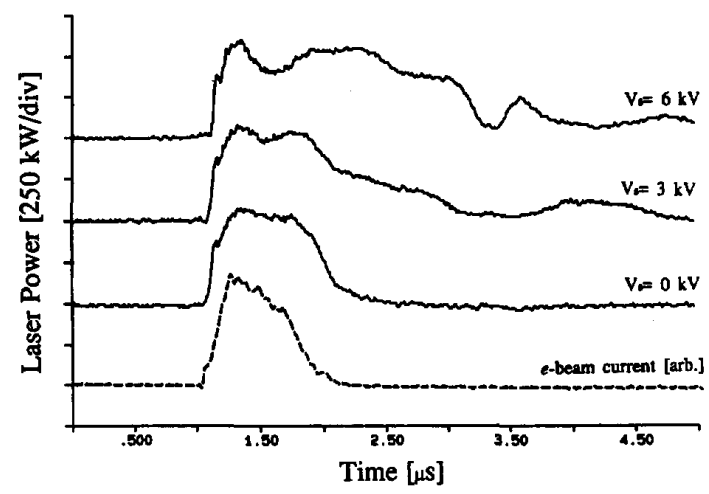

Fig. 6. Laser waveforms for different sustainer voltages, $\mathbf{P}=\mathbf{3} \mathrm{bar}, j_{\mathrm{hi}}=$ $0.9 \mathrm{~A} / \mathrm{cm}^{2}$.

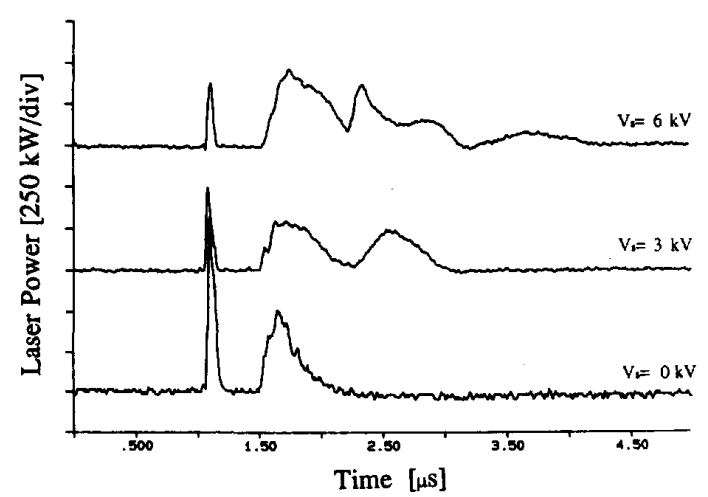

Fig. 7. Laser waveforms for different sustainer voltages, $\mathbf{P}=3 \mathrm{bar}, j_{\mathrm{hi}}=$ $2.7 \mathrm{~A} / \mathrm{cm}^{2}$.

which results from this pure $e$-beam excitation $\left(V_{\text {sust }}=0\right.$ $\mathrm{kV})$ : it follows the $e$-beam waveform. Applying the discharge by charging the sustainer capacitor to $3 \mathrm{kV}$ and 6 $\mathrm{kV}$, respectively, results in the upper two waveforms. Lasing is now observed during a discharge duration of typically $2.5 \mu \mathrm{s}$. A small decrease in laser power is observed $0.5 \mu \mathrm{s}$ after the beginning of the $e$-beam caused by ECM by discharge electrons.
In Fig. 7 the $e$-beam current density is increased to 2.7 A $/ \mathrm{cm}^{2}$. The $e$-beam waveform is the same as in Fig. 6 and is omitted. ECM by this high $e$-beam current density inhibits lasing during the first $0.5 \mu \mathrm{s}$ apart from a spike at the beginning of the $e$-beam. Lasing can start again when the fractional ionization $f$ has dropped to below a specific value $\left(f=n_{e} / N \simeq 2-8 \cdot 10^{-6}\right.$ in the case of $e$-beam excitation only, [3]) which results in the cessation of ECM. Population inversion is reestablished then by selective depopulation of the lower laser levels of xenon by collisions with argon. From Fig. 7 it is clear that the application of an electrical discharge results in increased laser action only under the condition that the fractional ionization $f$ has dropped to below the critical value.

On one hand side the discharge increases the inversion production, whereas on the other hand, it is expected that the resulting increased electron density will have a diminishing effect on the laser action due to ECM. Note the proportionality between the specific laser energy and the $e$-beam current density in Figs. $3-5$ in the case of pure $e$-beam pumping. Without the discharge a specific laser energy of $5 \mathrm{~J} / \mathrm{I}$ is obtained at $\mathbf{P}=7$ bar which is the highest reported specific laser energy of a pure $e$-beam excited xenon laser. Previously, we reported a maximum of $1.6 \mathrm{~J} / 1$ at $\mathbf{P}=4 \mathrm{bar}$ and $j_{\mathrm{hi}}=0.9 \mathrm{~A} / \mathrm{cm}^{2}$. The present increase of $j$ thus allows the use of higher pressures which results in a better laser performance.

In Fig. 5 at $\mathbf{P}=9$ bar at an optimum specific discharge energy deposition of about $340 \mathrm{~J} / 1$ the highest specific laser energy of $15 \mathrm{~J} / 1$ is obtained using $j_{\mathrm{hi}}=2.7 \mathrm{~A} / \mathrm{cm}^{2}$. At the lowest pressure of 3 bar an efficiency of $7-8 \%$ averaged over the complete discharge pulse is found. This efficiency is calculated by dividing the specific laser energy (minus the contribution of the $e$-beam) by the deposited specific discharge energy. At $\mathbf{P}=9$ bar this averaged efficiency drops to $4 \%$ because at this high pressure the 6s metastable xenon atoms decay rapidly after the $e$-beam duration.

The intrinsic efficiency is now defined during the time that the $e$-beam and discharge are simultaneously present. Since quasi-stationary laser action at high performance is obtained under those conditions the intrinsic efficiency is a realistic quantity for designing future devices. The intrinsic efficiency is calculated by dividing the laser power (minus the pure $e$-beam contribution) by the deposited discharge power in an interval of $0.25 \mu \mathrm{s}$ starting $0.5 \mu \mathrm{s}$ after the onset of the $e$-beam. In Fig. 8 the laser power and the intrinsic efficiency are both plotted against the discharge power for $\mathbf{P}=9$ bar. It is observed that the intrinsic efficiency can be $7 \%$ at this pressure, in agreement with [6].

\section{B. E/N Dependency on the e-Beam Current Density}

To obtain some insight in the discharge physics the peak discharge current is plotted as a function of the sustainer charging voltage for different $e$-beam current densities in Fig. 9 using a gas pressure of 7 bar. In order to be able 




Fig. 8. Laser power and intrinsic efficiency versus deposited power at 9 bar, $j_{\mathrm{hi}}=2.7 \mathrm{~A} / \mathrm{cm}^{2}$.

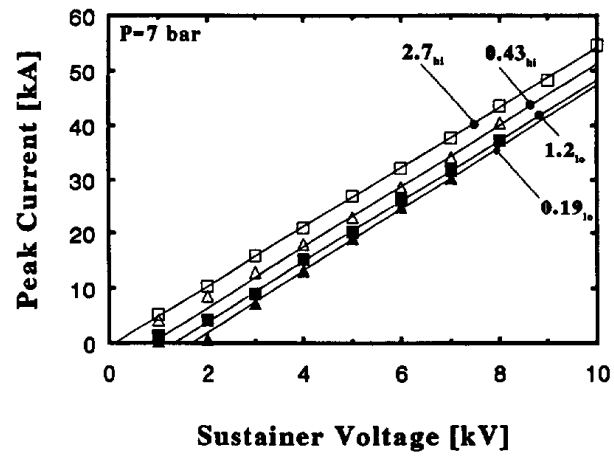

Fig. 9. Peak discharge current versus sustainer voltage at 7 bar for different $e$-beam parameters.

to observe the start-up of the discharge for low sustainer voltages the spark gap in the sustainer circuit has been removed. From Fig. 9 we observe that for sustainer charging voltages of $4 \mathrm{kV}$ and above all measured peak discharge currents show a linear dependency on the charging voltage. The slopes of these lines are independent of the $e$-beam current density and correspond to a dynamical resistance of $0.17 \Omega$. This resistance at peak current is found to be constant in the complete pressure range of 1-9 bar. From the calculated value of the circuit self-inductance and the known value of the capacitance $C$ the calculated impedance is: $\sqrt{L / C} \simeq 0.14 \Omega$. The discharge pulse duration is given by $\tau=\pi \sqrt{L C}=2.3 \mu \mathrm{s}$ (the measured values are 2.3-2.5 $\mu \mathrm{s}$ ). Clearly, the discharge parameters are governed by the self-inductance and the sustainer capacitance.

We extrapolate in Fig. 9 each one of the lines towards zero current, the intersection with the voltage axis results in the voltage we call the glow voltage. It accelerates the secondary electrons produced by the $e$-beam towards the discharge anode resulting in electron impact ionization of the active medium. From the glow voltage the glow electrical field $E_{\text {glow }}$ is found by dividing by the discharge electrode separation distance.

In Table II, $E_{\text {glow }}$ values are given as a function of the pressure, the $e$-beam energy and the $e$-beam current den-
TABLE II

$E_{\text {glow }}$ as a Function of the $e$-Beam Parameters and the Gas Pressure

\begin{tabular}{|c|c|c|c|c|c|c|}
\hline \multicolumn{7}{|c|}{ Glow Electric Field $[\mathrm{kV} / \mathrm{cm}]$} \\
\hline \multirow{2}{*}{$\begin{array}{c}\text { Pressure } \\
\text { [bar] }\end{array}$} & \multicolumn{3}{|c|}{$j_{\mathrm{lo}}\left[\mathrm{A} / \mathrm{cm}^{2}\right]$} & \multicolumn{3}{|c|}{$j_{\mathrm{hi}}\left[\mathrm{A} / \mathrm{cm}^{2}\right]$} \\
\hline & 0.19 & 0.40 & 1.20 & 0.43 & 0.90 & 2.70 \\
\hline 1 & 0.15 & 0.11 & 0.08 & 0.10 & 0.07 & 0.01 \\
\hline 3 & 0.53 & 0.43 & 0.26 & 0.35 & 0.28 & 0.01 \\
\hline 5 & 0.96 & 0.73 & 0.45 & 0.64 & 0.40 & 0.03 \\
\hline 7 & 1.44 & 1.12 & 0.68 & 0.92 & 0.60 & 0.07 \\
\hline 9 & - & - & - & - & - & 0.12 \\
\hline
\end{tabular}

sity. The glow fields $E_{\text {glow }}$ are found to increase almost linearly with pressure for each $e$-beam current density. Further $E_{\text {glow }}$ decreases with increasing ionization by the $e$-beam.

The current in the sustainer circuit depends only on the sustainer charging voltage and the circuit parameters $L$ and $C$. Its variations do not depend on the discharge itself. The gas resistance $\mathbf{R}_{\text {gas }}$ is very low (typ. a few $m \Omega$ at a gas pressure of 1 bar) and found to be proportional to the pressure. The voltage drop across the electrodes is proportional to the pressure and more or less independent on the current. Thus, $E / N$ is constant, in agreement with our observations.

\section{Discussion}

The present model that describes the power deposition of an electrical discharge under optimized excitation conditions is as follows:

The electrical power $P$ deposited in the discharge is given by:

$$
P=j \cdot E \quad\left[\mathrm{~W} / \mathrm{cm}^{3}\right]
$$

with $j$ the current density in $\left[\mathrm{A} / \mathrm{cm}^{2}\right]$ and $E$ the electrical field in $[\mathrm{V} / \mathrm{cm}]$.

The current density $j$ in (1) can be expressed as:

$$
j=e \cdot n_{e} \cdot v_{d}\left[\mathrm{~A} / \mathrm{cm}^{2}\right]
$$

in which the drift velocity $v_{d}$ in $[\mathrm{cm} / \mathrm{s}]$ is a function of $E / N[18]$.

The fractional ionization under optimized excitation conditions is defined as:

$$
f=n_{e} / N
$$

with $N$ the gas number density in $\left[\mathrm{cm}^{-3}\right]$.

The optimized power deposition $P_{\text {opt }}$ can now be written as:

$$
P_{\mathrm{opt}}=e \cdot E / N \cdot v_{d} \cdot f \cdot N^{2} \quad\left[\mathrm{~W} / \mathrm{cm}^{3}\right]
$$

Since the $E / N$-value is constant and consequently $v_{d}$ too [18] it is seen that the optimum deposited power is proportional to the square of the gas pressure. From Figs. 25 it follows that for both $e$-beam energies the optimum discharge energy deposition increases from $40 \mathrm{~J} / 1$ at $3 \mathrm{bar}$ (Fig. 2) to $340 \mathrm{~J} / 1$ at 9 bar (Fig. 5) thus almost proportional to $N^{2}$ in agreement with (4). 


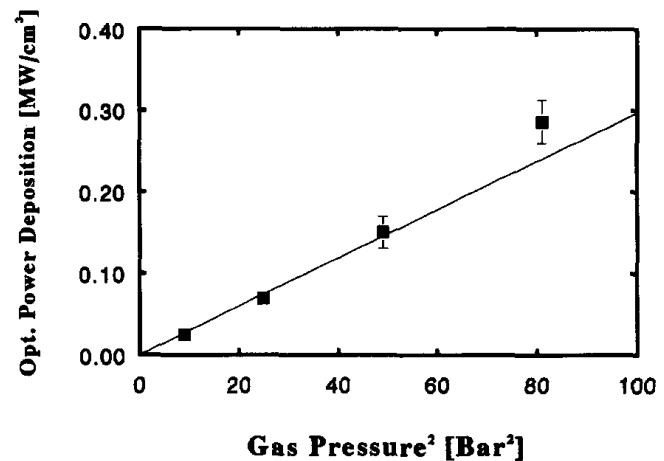

Fig. 10. Pressure dependency of the measured optimal discharge power deposition. The solid line is the theoretical curve from (4).

The fractional ionization $f$ is calculated from the electron density $n_{e}$ using (2) and (3). The electron drift velocity in (2) is taken from [18] using the measured value of the reduced electrical field $E / N$. Both $E / N$ and the current density are taken during optimized excitation conditions: about $0.5 \mu \mathrm{s}$ after the onset of the $e$-beam using the sustainer voltage which results in the maximal laser energy. An optimal fractional ionization $f$ of about 3.5-4.0 $\times 10^{-5}$ is found for all experimental conditions. If the discharge current density is increased further the corresponding $f$-value becomes higher than its optimal value and consequently the laser performance is reduced.

On the other hand, in a pure $e$-beam pumped xenon laser an $f$-value of $2-8 \times 10^{-6}$ is found [3]. This difference may be understood by the fact that the electron energy distribution is very different in an $e$-beam and thus a different quenching mechanism is expected. At a pressure of 9 bar, (4) is evaluated numerically using the fractional ionization value of $3.5-4 \times 10^{-5}$ found above. The electrode voltage is $1.83 \mathrm{kV}$, resulting in $E / N=0.41 \times$ $10^{-17} \mathrm{Vcm}^{2}$. From [18] the corresponding drift velocity $v_{d}$ is $2.3 \times 10^{5} \mathrm{~cm} / \mathrm{s}$. Using these values the optimum power deposition is calculated to be $0.28 \mathrm{MW} / \mathrm{cm}^{3}$. The measured power deposition in the quasi-stationary regime is $0.24 \mathrm{MW} / \mathrm{cm}^{3}$. In Fig. 10 the measured optimum discharge power is plotted against the pressure squared, resulting in a linear relationship as predicted by (4). The solid line represents the theoretical curve, given by (4) with $v_{d}=2.3 \times 10^{5} \mathrm{~cm} / \mathrm{s}, E / N=0.41 \mathrm{Vcm}^{2}$ and $f=$ $3.7 \times 10^{-5}$. The value of $E / N$ is constant resulting in a constant drift velocity. Therefore, the observed linear relationship in Fig. 10 confirms the constant value of the fractional ionization at which the laser shows an optimal performance: $f=4 \times 10^{-5}$.

\section{Conclusions}

Increasing the $270 \mathrm{keV} e$-beam current density to 2.7 $\mathrm{A} / \mathrm{cm}^{2}$ has resulted in the highest reported specific energy of $15 \mathrm{~J} / 1$ in an $e$-beam sustained discharge xenon laser at a gas pressure of 9 bar. In the case of pure $e$-beam excitation the highest reported specific energy is $5 \mathrm{~J} / 1$ at a gas pressure of 7 bar. The enhancement of the laser energy so far by adding the discharge to the $e$-beam excitation is thus about three times. An optimum fractional ionization factor $f=3.5-4 \times 10^{-5}$ is found, independent of the $e$-beam parameters. Quasi-stationary laser action during the simultaneous presence of $e$-beam and discharge is observed with an intrinsic efficiency of 7-8\%. A simple discharge model predicts the optimal discharge energy deposition $P_{\mathrm{opt}}=3.0 \mathrm{kWcm}^{-3} \mathrm{bar}^{-2}$, in good agreement with the experimental results.

\section{REFERENCES}

[1] W. J. Witteman, The $\mathrm{CO}_{2}$ Laser. Berlin: Springer Verlag, 1987, p. 193.

[2] M. Ohwa, T. J. Moratz, and M. J. Kushner, “Excitation mechanisms of the electron-beam-pumped atomic xenon (5d-6p) laser in $\mathrm{Ar} / \mathrm{Xe}$ mixtures," J. Appl. Phys., vol. 66, pp. 5131-5145, 1989.

[3] P. J. M. Peters, Y. F. Lan, M. Ohwa, and M. J. Kushner, "Impact of electron collision mixing on the delay times of an electron beam excited atomic xenon laser," IEEE J. Quantum Electron., vol. 26, pp. 1964-1970, 1990.

[4] L. N. Litzenberger, D. W. Trainor, and M. W. McGeoch, "A $650 \mathrm{~J}$ e-beam atomic xenon laser," IEEE J. Quantum Electron., vol. 26, pp. 1668-1675, 1990.

[5] R. L. Watterson and J. H. Jakob, "Measurements of intrinsic efficiency and parameters of an electron beam pumped ArXe laser," IEEE J. Quantum Electron., vol. 26, pp. 417-422, 1990.

[6] H. Botma, P. J. M. Peters, and W. J. Witteman, "Intrinsic efficiency and critical power deposition in the e-beam sustained Ar: Xe laser," Appl. Phys. B, vol. 52, pp. 277-280, 1991.

[7] L. A. Newman and T. A. DeTemple, "High pressure infrared Ar-Xe laser system: Ionizer-sustainer mode of operation," Appl. Phys. Lett., vol. 27, pp. 678-680, 1975.

[8] N. G. Basov, V. V. Baranov, A. Y. Chuganov, V. A. Danilychev, V. A. Danilychev, A. Y. Dudin, I. V. Kholin, N. N. Ustinovskii, and D. A. Zayarnyi, " $60 \mathrm{~J}$ quasistationary electroionization laser on Xe atomic metastables," IEEE J. Quantum Electron., vol. 21, pp. $1756-1760,1985$.

[9] A. Suda, B. L. Wexler, K. J. Riley, and B. J. Feldman, "Characteristics of the high-pressure Ar-Xe laser pumped by an electron beam and an electron-beam sustained discharge," IEEE J. Quantum Electron., vol. 26, pp. 911-921, 1990.

[10] G. A. Batyrbekov, E. G. Batyrbekov, V. A. Danilychev, A. B. Tleuzhano, and M. U. Khasenov, "Electric-discharge xenon laser with weak external ionization," Sov. J. Quantum Electron., vol. 19, pp. 1393-1395, 1989.

[11] G. G. Baitsur, V. V. Kralin, and K. N. Firsov, "High pressure ArXe laser pumped by a self-sustained volume discharge," Sov. J. Quantum Electron., vol. 20, pp. 1447-1448, 1990.

[12] J. E. Tucker, B. L. Wexler, B. J. Feldman, and T. McClelland, "High-pressure infrared xenon laser with $\mathrm{X}$-ray preionization," IEEE Photon. Tech. Lett., vol. 1, pp. 193-195, 1989.

[13] Ch. K. Rhodes, Excimer Lasers (2nd ed.). Berlin: Springer Verlag, 1984.

[14] N. G. Basov, V. V. Baranov, V. A. Danilychev, A. Yu. Dudin, D. A. Zayamyi, A. V. Rzhecskii, N. N. Ustinovskii, I. V. Kholin, and A. Yu. Chugunov, "Possibility of construction of a pulse-periodic large-volume electron-beam-controlled laser utilizing infrared transitions in the Xe atom and characterized by a specific output power of 0.5-1 W $/ \mathrm{cm}^{3}$," Sov. J. Quantum Electron., vol. 16, pp. 1008$1009,1986$.

[15] S. G. Perlov, N. N. Ustinovskii, I. V. Kholin, and A. Yu. Chugunov, "Atmospheric-pressure electron-beam-controlled Ar-Xe laser," Sov. J. Quantum Electron., vol. 19, pp. 733-734, 1989.

[16] D. A. Zayarnyi, A. G. Korolev, N. N. Sazhina, N. N. Ustinovskii, and I. V. Kholin, "Influence of the pump power on the spectral and time characteristics of an Ar-Xe laser," Sov. J. Quantum Electron., vol. 21 , pp. 488-494, 1991

[17] J. J. Berger and S. M. Seltzer, Nucl. Sci. Series no. 10, NAS-NRC pub. $113,1964$.

[18] E. Richley, "Extending the calculation of electron velocity distribution functions for electric discharges to large values of $\mathrm{E} / \mathrm{N}$," $J$. Appl. Phys., vol. 71, pp. 4190-4195, 1992. 


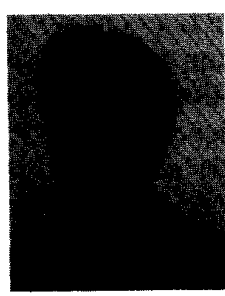

Hako Botma was born in Nijmegen, The Netherlands, on July 6,1959 . He received his M.S degree from the Catholic University of Nijmegen in 1988 and his Ph.D. degree in 1993 from the University of Twente, Enschede.

His interests include gas-laser excitation mechanisms, laser kinetics, and laser spectroscopy. He currently researches VUV solid state lasers.

$\mathrm{He}$ is a member of the Dutch Physical Society NNV.

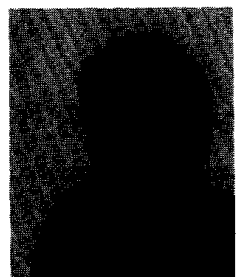

Peter J. Peters was born in Meerlo, The Netherlands, on November 5,1945 . He received the M.S. degree from the Catholic University of Nijmegen in 1975 and the Ph.D. degree in 1981 from the University of Twente, Enschede, The Netherlands, all in physics. In 1981 he joined the staff of the Quantum Electronics group. He is now an associate Professor with the University of Twente and has conducted research on TEA CO discharge lasers, various excimer lasers, and atomic rare-gas lasers.

W. J. Witteman, photograph and biography not available at the time of publication. 